\title{
A REMARKABLE SIMPLE CLOSED CURVE: REVISITED
}

\author{
O. G. HARROLD
}

\begin{abstract}
It is shown that the pathology of R. H. Fox's remarkable simple closed curve is in a sense explained below more complicated than that of some examples of the well-known Fox-Artin paper.
\end{abstract}

A classical example of Fox-Artin shows that the union of two tamely embedded arcs whose intersection is a common end-point may be wildly embedded [3, Example 1.4]. Arcs formed in this way are called mildly wild. A classification theorem exists for such sets if the union is locally peripherally unknotted, LPU, also called Wilder arcs [4]. While the concepts of LPU and LU [7] have served to classify the local pathology of the examples of [3], a more delicate invariant seems necessary to relate the embedding of [5] to those in [3]. There is the possibility that an appropriately chosen arc from "The remarkable simple closed curve" might be "less wildly" embedded than the arcs in [3]. This note dispells such feasability. To make this more precise a new embedding condition is introduced related to LPU. First, however, we review Fox's concept of almost unknotted.

A simple closed curve $\Gamma$ in three-space $\Re^{3}$ is called almost unknotted if there is a point $p$ and a neighborhood $U$ of $p$ such that for any neighborhood $V$ of $p$ there is a homeomorphism $\phi$ of $\Re^{3}$ on $\Re^{3}$ such that

(i) $\phi$ is the identity on $V$,

(ii) $\phi \mid \Gamma \backslash U$ is a subset of a plane.

It is clear that if $q$ is another point and $\Gamma$ has the same property at $q$, then $\Gamma$ is unknotted.

There is a local property suggested by the above. An arc $X$ is called locally almost unknotted at $p$ if for some neighborhood $U$ of $p$, no matter how small a neighborhood $V$ of $p$ is chosen, there is a neighborhood $W$ of $p$ and a homeomorphism $\phi$ of $\Re^{3}$ on $\Re^{3}$ such that

(i) $\phi=$ identity on $W$ and

(ii) $\phi \mid U \backslash V$ is a subset of a plane. We abbreviate this property by LAU (locally almost unknotted).

Before introducing the next definition recall the definition of local peripheral unknottedness for a 1-manifold in $\Re^{3}$ [7]. Let $p$ be an interior point (boundary point) of $X$ and $\varepsilon>0$. It is required that there be a topological 2-sphere $K$ whose interior contains $p$ such that

Received by the editors February 8, 1980. Presented to the Florida section of the Mathematical Association of America at the annual meeting at Jacksonville University, Jacksonville, Florida, March 7, 1980.

1980 Mathematics Subject Classification. Primary 55A30, 57A40. 
(i) $\operatorname{diam} K<\varepsilon$,

(ii) card $K \cap X=2$ or 1 according as $p$ is an interior or boundary point of $X$. See [3, Examples 1.1 or 1.2] for the failure of this property.

A 1-manifold $X \subset \Re^{3}$ is called weakly peripherally unknotted at $p$ if for each $\varepsilon>0$ there is a homeomorphism $h_{\varepsilon}$ of $\Re^{3}$ on $\Re^{3}$ such that

(0) $h_{e}(p)=p$,

(i) $\operatorname{diam} h_{\varepsilon}^{-1}(K)<\varepsilon$,

(ii) card $K \cap X=2$ or 1 according as $p$ is an interior or boundary point of $X$. We abbreviate this property by WPU. Note "The remarkable simple closed curve" has this property at each point.

If one may take $h_{e}=$ identity for all $\varepsilon>0$, this becomes LPU.

As a preliminary to the main result (Theorem 2) we have the following.

THEOREM 1. Let $X$ be an arc that is locally tame modulo $p, p$ an end-point of $X$. Then $X$ is LAU at $p$.

Proof. If the penetration index of $X$ at $p$ is 1 , a sequence of space homeomorphisms can be defined carrying $X$ onto an interval. Hence $X$ is tame. In general, by the smoothing techniques of [1] or [9], $X \backslash p$ may be taken as locally polyhedral. If the other end-point of $X$ is $q$, let $X$ be ordered from $q$ to $p$. Also, let $F(p, \varepsilon)$ denote the surface of a sphere of radius $\varepsilon$, centered at $p$. Let $Y_{\varepsilon}$ denote the subarc of $X$ from $q$ to $t_{e}$, the last point on $X$ in the assigned order. Then $Y_{e}$ is a finite polygonal arc and by elementary means (see Graueb [6]) can be straightened out to a segment $Y_{\varepsilon}^{\prime}$ leaving a neighborhood of $p$ pointwise fixed. Denote this semilinear homeomorphism by $f_{e}$. Let a neighborhood of $p$ that is pointwise fixed by $f_{e}$ be $Z_{e}$. We note $X$ becomes $f_{\varepsilon}(X)$ and the part of $f_{\varepsilon}(X)$ exterior to $F(p, \varepsilon)$ is a segment and hence lies in a plane. If $\varepsilon^{\prime}>\varepsilon, F\left(p, \varepsilon^{\prime}\right)$ meets $f_{e}(X)$ in at most one point. Then $K=$ $f_{\varepsilon}^{-1}\left[F\left(p, \varepsilon^{\prime}\right)\right]$ is a topological 2-sphere that meets $X$ in a single point if $\varepsilon^{\prime}-\varepsilon$ is sufficiently small. Since the points of $Z_{e}$ have remained pointwise fixed, $X$ is LAU at $p$.

Remark 1. The segment $Y_{\varepsilon}^{\prime}$ referred to above may be taken to lie on a line through $p$.

REMARK 2. Among the arcs locally tame modulo an end-point, the LAU condition is no further restriction of the embedding.

REMARK 3. Let $X$ be a union of two $\operatorname{arcs} X^{\prime}$ and $X^{\prime \prime}$ meeting at $p$. Assume $X^{\prime} \backslash p$ and $X^{\prime \prime} \backslash p$ are locally tame. If $X$ has penetration index $=2$ at $p$, then $X^{\prime}$ and $X^{\prime \prime}$ are each tame and $X$ is at most mildly wild [8]. Applying the above calculations to $X^{\prime}$ and $X^{\prime \prime}$, we see that the hypothesis that $X$ be LAU at $p$ implies that the homeomorphisms used in straightening $X^{\prime}$ and $X^{\prime \prime}$ be consistent, i.e. there is a single homeomorphism that straightens each of $X^{\prime}, X^{\prime \prime}$ except for a neighborhood of $p$. This will be taken as our definition of LAU at an interior point of an arc below.

THEOREM 2. Among the mildly wild arcs, LAU and WPU are equivalent properties.

Proof. Let $X=X^{\prime} \cup X^{\prime \prime}$ be mildly wild, where $X^{\prime} \cap X^{\prime \prime}=\{p\}, X^{\prime}, X^{\prime \prime}$ are 
tame and $X$ is LAU at $p$. Given $\varepsilon>0$, define $U=S(p, \varepsilon) \cap X$. Let $V$ be the component of $S(p, \varepsilon / 3) \cap X$ determined by $p$. By the LAU property, there is a neighborhood $W$ of $p$ and a homeomorphism $\xi$ of $\mathbf{R}^{3}$ on $\mathbf{R}^{3}$ such that

(i) $\xi \mid W=$ identity,

(ii) $\xi(U \backslash V)$ is a subset of plane $\pi$. Diminishing $W$ if necessary we assume $W=S(p, \delta)$ and for all $x$ in $W$ the arc $x p$ has a diameter $<\varepsilon / 3$. If $q_{-1}$ and $q_{+1}$ are the end-points of $X$, let $X$ be reparametrized so that $q_{-1}$ corresponds to $t=-1$, $p$ to $t=0$ and $t=+1$ to $q_{1}$. Let $A$ denote the first component of $\left(q_{-1} p\right) \cap \pi$ with one boundary component on $F\left(p, \varepsilon^{\prime}\right), \varepsilon^{\prime}>\varepsilon$, and one on $F(p, \varepsilon)$. Let $B$ denote the last component of $\left(p q_{1}\right) \cap \pi$ with one boundary component on $F(p, \varepsilon)$ and one on $F\left(p, \varepsilon^{\prime}\right)$. All other components of $\pi \cap(X \backslash W)$ can be pushed into $S(p, \varepsilon) \backslash W$ without moving the points of $W$ by a homeomorphism $\zeta_{2}$. The set $\left\{S\left(p, \varepsilon^{\prime}\right) \backslash S(p, \varepsilon)\right\} \cap \pi$ is an annulus with two components of

$$
\left\{\left\{S\left(p, \varepsilon^{\prime}\right) \backslash S(p, \varepsilon)\right\}\right\} \cap X,
$$

call them $A$ and $B$, stretching from $F\left(p, \varepsilon^{\prime}\right)$ to $F(p, \varepsilon)$. Let $H$ be a simple closed curve in this annulus piercing both of $A$ and $B$ just once. Then define $K=H \times$ $[-\varepsilon,+\varepsilon] \cup($ Int $H \times \varepsilon) \cup\{($ Int $H) \times-\varepsilon\}$. Clearly $K$ is a topological 2-sphere containing $p$ in its interior and $K \cap X=$ a pair of points, one on $A$, one on $B$ and diam $K<2 \varepsilon^{\prime}+2 \varepsilon \leqslant 4 \varepsilon^{\prime}$ (using a rectangular metric).

By construction, diam $K<3 \varepsilon$ if $\varepsilon^{\prime}-\varepsilon$ is sufficiently small. Taking $h=\left(\zeta_{2} \zeta\right)^{-1}$ we have

(0) $h(p)=p$,

(i) $\operatorname{diam} K<3 \varepsilon$,

(ii) card $K \cap h^{-1}(X)=2$, i.e., $X$ is WPU at $p$.

In the converse direction, suppose $X=A \cup B$, where $A$ and $B$ are tame and $A \cap B=\{p\}$ is a common end-point. Assuming $X$ is WPU at $p$ we want to prove $X$ is LAU at $p$. Given $\varepsilon>0$, there is a topological 2-sphere $K$ containing $p$ in its interior and a homeomorphism $h$ of $\mathbf{R}^{3}$ on $\mathbf{R}^{3}$ such that

(0) $h(p)=p$,

(i) $\operatorname{diam} h^{-1}(K)<\varepsilon$,

(ii) $K \cap X=a \cup b, a \in A$ and $b \in B$.

Let $A_{1}$ denote the component of $A^{\prime} \backslash a$ not containing $p$ and $B_{1}$ the component of $B^{\prime} \backslash b$ not containing $p$. Then $A_{1}$ and $B_{1}$ are tame disjoint arcs defined by the WPU property. By the smoothing techniques of [1] or [9] we may choose $\bar{A}_{1}$ and $\bar{B}_{1}$ as polygonal arcs. Again, by elementary techniques $\bar{A}_{1} \cup \bar{B}_{1}$ may be flattened out into a plane leaving a neighborhood $S$ of $p$ pointwise fixed by a homeomorphism $g$.

If $U$ is any neighborhood of $p$ large enough to contain $X \backslash g\left(A_{1} \cup B_{1}\right)$, then given any $V(p) \subset U$ there is a $W(p) \subset V \subset S$ so that

(i) $g \mid W=$ identity,

(ii) $g \mid U \backslash V$ is a subset of a plane.

This serves in the definition of LAU for $X$ at $p$.

TheOREM 3. Let $\Gamma$ again denote "The remarkable simple closed curve." Then $\Gamma$ fails to be either locally unknotted $(L U)$ or locally peripherally unknotted $(L P U)$ at $p$. 
Proof. Suppose $\Gamma$ is LU at $p$. Then there is a disk $D$ containing a neighborhood of $p$ in $\Gamma$. Since $\Gamma$ is locally tame $\bmod p$, there is no loss in choosing $D$ locally polyhedral $\bmod p$. Let $Y=A \cup B$ be a neighborhood of $p$ in $\Gamma$ where $A$ is a straight line interval with $p$ as an end-point and $B$ the closure of the complement in $Y$. Then $A$ and $B$ are equivalently embedded in $\mathbf{R}^{3}$ by Theorem 5 of [2]. Hence $A$ and $B$ are both tame. The existence of $D$ means $A \cup B$ has the A1P at $p$. Thus $A \cup B$ is tame by [8], a contradiction. Hence $\Gamma$ is locally knotted at $p$ (i.e. no such $D$ exists).

If $\Gamma$ were LPU at $p, \Gamma$ would be expressible as a union of two nonoverlapping arcs $q a p, q b p$ denoted by $A, B$, respectively. At least one of $A, B$ is tame, say $A$. There is a disk $D$ whose boundary contains $A$ and $D$ may be chosen tame. By choosing $D$ carefully we can arrange that $D \cap B=\{p\}$. Since $\Gamma$ is LPU at $p, B$ is LPU at $p$. Hence $B$ is tame. It follows that $A \cup B$ is part of a tame arc and hence tame, a contradiction.

Thus, the embedding of $\Gamma$ at $p$ is more complicated than that of either 1.2 or 1.4 of [3]. $\Gamma$ is not a union of two tame nonoverlapping arcs but is a countable union of such tame nonoverlapping arcs.

\section{REFERENCES}

1. R. H. Bing, Locally tame sets are tame, Ann. of Math. 59 (1954), 145-158.

2. P. H. Doyle and J. G. Hocking, Some results on tame disks and spheres on $E^{3}$, Proc. Amer. Math. Soc. 11 (1960), 832-836.

3. R. H. Fox and E. Artin, Some wild cells and spheres in three-space, Ann., of Math. 49 (1948), 979-990.

4. R. H. Fox and O. G. Harrold, The wilder arcs. Topology of 3-manifolds and related topics (Proc. The Univ. of Georgia Institute, 1961), Prentice-Hall, Engelwood Cliffs, N.J., 1962, pp. 184-185.

5. R. H. Fox, A remarkable simple closed curve, Ann. of Math. 50 (1949), 264-265.

6. W. Graeub, Die semilinear Abbildungen, S.-B. Heidelberger Akad. Wiss. Math.-Nat. Kl. 1950, pp. 205-272.

7. O. G. Harrold, Locally tame curves and surfaces in three-dimensional manifolds, Bull. Amer. Math. Soc. 63 (1957), 302.

8. __ An Additive Index Theorem for certain wildly embedded curves and surfaces (Proc. Conf. Geometric Topology), Academic Press, New York, 1979.

9. E. E. Moise, Affine structures in 3-manifold. VIII, Ann. of Math. 59 (1954), 159-170.

Department of Mathematics, Florida State University, Tallahassee, Florida 32306 